\title{
Controversias en torno a las salidas profesionales del nuevo grado de Protocolo y su relación con las otras carreras de Comunicación
}

\author{
Fernando RAMOS FERNÁNDEZ \\ Universidad de Vigo \\ ferramos@telefonica.net
}

Recibido: 03/10/2012

Aceptado: 23/01/2013

\begin{abstract}
Resumen
La defensa por parte de sus promotores del nuevo grado de Protocolo que actualmente ofrecen dos universidades españolas provoca viscerales reacciones cuando cualquiera quiere plantear un mero análisis crítico sobre la incongruencia que supone que la nueva carrera, que se dijo venía a ocupar un espacio propio colmando el deseo de amplias masas de profesionales, ofrezca repetidamente las mismas salidas que ya cubren otros estudios de Comunicación, de mayor abolengo y experiencia. Y ello pese a que contraviene las propias previsiones de la ANECA de no poner en el mercado titulaciones cuyas salidas profesionales ya estuvieran atendidas por otros estudios. El riesgo de confrontación entre estudios del ámbito de la comunicación, la feroz competencia o la frustración son riesgos apreciables que, sin embargo, parecen dispuestos a no afrontarse.
\end{abstract}

Palabras clave: ANECA. Protocolo. Relaciones Públicas. Periodismo. Salidas profesionales. Mercado

\section{Controversies Surrounding the Career of the New Degree of Protocol and its Relationship with the other Races of the Communication}

\begin{abstract}
The defense by its promoters of the new degree of Protocol currently offering two Spanish universities provokes visceral reactions when anyone wants to raise a mere critical analysis of incongruity is that the new race, which he said came to occupy its own space filling the desire for broad masses of professionals, offering repeatedly the same outlets that already cover other communication studies, most pedigree and experience. And despite the fact that contravenes the provisions of the ANECA own not to put on the market whose career qualifications were already served by other studies. The risk of confrontation between studies in the field of communication, fierce competition or frustration are significant risks, however, seem willing to not addressed.
\end{abstract}

Keywords: ANECA. Protocol. Public Relations. Journalism. Career. Market

\section{Referencia normalizada}

RAMOS FERNÁNDEZ, Fernando (2013): “Controversias en torno a las salidas profesionales del nuevo grado de Protocolo y su relación con las otras carreras de Comunicación”. Estudios sobre el mensaje periodístico. Vol. 19. Núm. especial abril, págs.: 935-943. Madrid, Servicio de Publicaciones de la Universidad Complutense.

Sumario: 1.La ubicación de los estudios de Protocolo en la Universidad española. 2. La necesidad de poner orden en los estudios ofertados. 3. La solución del empleo en la Administración. 4. La situación profesional del Protocolo como actividad específica. 5. La controvertida coincidencia de salidas profesionales. 6. Conclusiones. 7. Referencias bibliográficas.

\section{La ubicación de los estudios de Protocolo en la Universidad española}

El Protocolo llevaba muchos años en la Universidad. Desde los pioneros cursos de la de Oviedo, es ya densa la producción de tesis doctorales, líneas de investigación, cursos y títulos propios, por no citar la interminable serie de master, cursos de especia- 
listas y otras diversas fórmulas tanto en las Universidades públicas como en las privadas.

En 2001, la Convención de Instituciones Europeas de Enseñanza Superior, con Relación a la aplicación del Plan Bolonia advirtió:

"Las titulaciones propuestas por los Estados habrán de responder a demandas sociales por lo que se hace necesario un análisis y definición de perfiles profesionales considerando, entre otros, las características del mercado laboral nacional y europeo, el parecer de las asociaciones profesionales y la experiencia acumulada por las propias universidades. [...] No procederá establecer un titulo oficial cuyos contenidos y efectos profesionales coincidan con otro".

Esa demanda social existía, sin duda alguna, por parte de los profesionales del Protocolo, sin conocida excepción, salvo quizá en el ámbito académico. Pero el simple cotejo de los vigentes planes de estudio y salidas profesionales de las dos Universidades (una privada y otra pública) que en estos momentos ofrecen el grado de Protocolo y Organización de Eventos nos depara la sorpresa de hallar, en ambos casos, repetidas coincidencias con salidas profesionales específicas que ya están cubiertas por otras carreras con mayor abolengo dentro de la familia de la Comunicación.

La Universidad Camilo José Cela (privada) ofrece estas salidas coincidentes en su web: Dirección y planificación estratégica y cuentas. Director de relaciones institucionales o de relaciones externas. Director de gabinete. Director de comunicación. Ejecutivo de cuentas en agencias de comunicación. Asesor de imagen. Planificadores de medios. Dirección de comunicación. Director/a de publicidad. Director/a de relaciones públicas.

Por su parte, la Universidad Miguel Hernández de Elche (pública) ofrece a sus futuros egresados varias salidas profesionales, agrupadas por área: Director de Comunicación, Jefe de Prensa, Jefe de Gabinete, Relaciones Públicas en empresas o instituciones, Secretario/a de Alta Dirección, Asesor de Imagen.

La aparición de la nueva carrera de Protocolo y Organización de Eventos produjo un estallido de alegría entre los profesionales de Protocolo, e incluso, desde algunos foros se puso en marcha una visible operación de marketing a fin de reclutar con rapidez alumnos con que alimentar las aulas de la primera universidad, privada, que se apresuró a ofrecer la nueva titulación. Dentro de esa política se animó a todos los profesionales a titularse en el nuevo grado, ofreciendo todo de facilidades e incluso pasarelas para permitir que nadie quedara fuera de la nueva carrera. Del mismo modo, se puso en marcha una campaña para reclamar un estatuto jurídico que reconociera al protocolo como una actividad profesional regulada y colegiada, siendo indispensable, según sus promotores, estar en posesión del grado (que en aquellos momentos sólo ofrecía una universidad) y debidamente colegiado.

$\mathrm{Ni}$ en periodismo, publicidad o las relaciones públicas, la titulación académica es un requisito imprescindible para el ejercicio profesional, aunque evidentemente capacite para el mismo. De hecho, es el la carrera de periodismo donde es más elevado el porcentaje de profesionales que poseen la licenciatura correspondiente. Por otro lado, en cuanto a la adscripción de los profesionales a un colegio (entidad de derecho público) o a una asociación (de derecho privado) son iguales las consecuencias prácticas, dado 
que al no requerirse titulación para el ejercicio, colegios o asociaciones no son otras cosas que entidades de afiliación voluntaria. El promedio de alumnos matriculados en los actuales estudios de comunicación en toda España oscila por curso entre 13.500 y 14.000, de los que cada año acaban la carrera alrededor de 4.500 a 5.000. De ellos, de tres a cuatro de cada diez son licenciados en publicidad y relaciones públicas.

La plena incorporación de los estudios de las Ciencias de la Comunicación, de la Información, a la Universidad, a partir del inicio de los años setenta supuso un paso decisivo hacia la consolidación de una materia específica, incorporada desde entonces a los planes de estudio de las carreras de la comunicación. Recuerda Fernández Areal (1977, Pág. 50) que la creación de las Facultades de Ciencias de la Información, que trató de dotar de contenido científico a los estudios que se consideran básicos para una potenciación de la profesión informativa, trajo como consecuencia, al estructurarse sus planes de estudio, la necesidad de enfrentarse con la metodología y docencia de la legislación de Prensa nacional, sin olvidar el Derecho comparado, -por su valor formativo y su eficacia práctica-, si se considera la movilidad innata de muchos de cuantos se dedican a la "peligrosa tarea" de informar.

Las investigaciones de Vivar, García, Abuín y Vinader (2010) sobre la situación de los estudios de comunicación en España frente al reto del Espacio Europeo de Educación Superior, han centrado la naturaleza del problema:

"Estos estudios incrementan su demanda año tras año. No obstante, ésta no va acompañada de una inserción laboral proporcional, lo que, unido a la profunda crisis por la que están atravesando los grupos de comunicación, está provocando una importante tasa de paro entre los licenciados en alguna de las tres titulaciones (tradicionales), que optan por buscar salidas profesionales alternativas."

\section{La necesidad de poner orden en los estudios ofertados}

Las conclusiones del documentado trabajo de Vivar, García, Abuín y Vinader (2010) sobre los estudios de comunicación en España deberían hacer reflexionar a la ANECA sobre alguna de sus políticas, tras analizar la situación de los titulados en Comunicación, junto con la implantación de las nuevas tecnologías en nuestro país y los planes de estudio que marcarán la oferta formativa de las disciplinas objeto de estudio.

La primera, que plenamente compartimos, es que la elevada demanda de las titulaciones en Comunicación propicia la promoción de una cantidad de licenciados que el mercado laboral no puede absorber, provocando que estos titulados desempeñen trabajos muy alejados de la preparación que han recibido. Es necesario, como se apunta, ajustar la formación de los titulados a las necesidades de los medios de comunicación, dominados por la llegada de las nuevas tecnologías que mueven unos volúmenes ingentes de negocio. Los planes de estudio deben tener en cuenta las necesidades de la industria donde se van a integrar los nuevos graduados. Conectar los planes de estudio a la nueva realidad tiene que convertirse en el punto de partida sobre el que cimentar la colaboración entre la Universidad y la empresa, para lograr estabilizar y potenciar un mercado incipiente de los contenidos digitales.

La estadística sobre el empleo de los profesionales de la comunicación en España ha tenido escasos referentes que puedan ser considerados fiables y propiamente cien- 
tíficos, a excepción del "Informe Anual sobre la Profesión Periodística" que edita la Asociación de la Prensa de Madrid. Para darse una idea de la crisis de empleo que afecta al sector de la comunicación (especialmente a tener en cuenta cuando se lanzan al mercado nuevos titulados que van a competir) en el mismo espacio con los ya presentes, cabe recodar que, según el mencionado informe, el aumento del número de periodistas desempleados ha pasado de 3.030 en el período de 2008-2009 a 6.500 parados en esta última encuesta, según Pedro Farias, profesor de la Universidad de Málaga y director del 'Informe Anual de la Profesión Periodística', quien subrayó que la cifra se elevará a 10.000 periodistas en paro al añadirle los que se gradúan cada año en las facultades de comunicación ${ }^{1}$.

Según datos de la propia ANECA, de las 17 comunidades autónomas del Reino de España, 11 cuentan con centros en los que se ofertan estudios de comunicación. En concreto, Cataluña (11), Madrid (8), Valencia (5), Castilla-León (5), Galicia (3), Andalucía (2), Murcia (2), País Vasco (1), Navarra (1), Extremadura (1) y Canarias (1). E1 60\% de los centros que imparten titulaciones de comunicación dependen de universidades públicas, mientras que el $40 \%$ de privadas. Publicidad y Relaciones Públicas pueden cursarse en 27 facultades.

Al igual que en Comunicación Audiovisual, el auge de los estudios de Publicidad y Relaciones Públicas en España se localiza fundamentalmente en los años 90. Es a partir de ese momento cuando se inicia un incremento constante en el número de facultades que pasan a impartir esta carrera, un aumento que en un principio está claramente liderado por las universidades privadas. Desde 1990 y hasta 2000, comienzan estos estudios en nueve centros privados por cinco públicos.

El reconocimiento por parte de la ANECA del Grado en la nueva carrera de Protocolo y Organización de Eventos fue saludada con gran entusiasmo y alborozo por los profesionales de este sector, si bien, la lectura con calma del desarrollo de los acontecimientos posteriores al pasado 30 de septiembre de 2010 -en que nace oficialmente la nueva carrera dentro de la familia de las Ciencias de la Comunicación- sigue mereciendo alguna reflexión para centrar el asunto, teniendo en cuenta que otras universidades están dispuestas a ofrecer también esta carrera.

No se puede ignorar, pues, la situación actual de los estudios de Relaciones Públicas, su historial, programas, itinerarios, especialización y salidas profesionales, así como de la oferta complementaria de estudios post grado o privados, que denotan una saturación de la oferta de estudios de Protocolo de muy diversa calidad y nivel. ${ }^{2}$.

1 Sucesivos Expedientes de Regulación de Empleo que continúan negociándose dos años después de que comenzara la crisis (Colpisa), recorte en los salarios de los colaboradores (El País), aumento de la carga de trabajo (El Mundo), dibujan el panorama del empleo para los periodistas hoy en día.

2 La calidad de los estudios depende de la solvencia y adecuación de los programas, del rigor y conocimiento de los profesores, de la seriedad a la hora de organizar la docencia y evaluar a los alumnos, que son eso ante todo, y no meros clientes a los que haya que asegurar una salida si no la merecen y saben ganarla. 


\section{La solución del empleo en la Administración}

Se han formulado diversas reflexiones sobre el futuro de los profesionales del Protocolo, especialmente en el ámbito de las administraciones públicas. Consecuentemente se concluye que, aparte de un reconocimiento oficial de la profesión, mediante alguna fórmula de estatuto oficial (del que carecen todos las demás profesiones de la Comunicación, por cierto, salvo la regulación de la cláusula de conciencia y el secreto profesional del periodista, constitucionalmente previstos), en el futuro, sea condición habilitante inexcusable disponer de un concreto título para acceder a determinados puestos en las instituciones oficiales (y queremos entender que en las privadas) para una función específicamente definida.

El vigente Estatuto de la Función Pública señala:

"El sistema de empleo público que permita servir adecuadamente a los ciudadanos es aquel que hace posible atraer los profesionales que la Administración necesita, que estimula a los empleados para el cumplimiento eficiente de sus funciones y responsabilidades, les proporciona la formación adecuada y les brinda suficientes oportunidades de promoción profesional, al tiempo que facilita una gestión racional y objetiva, ágil y flexible del personal, atendiendo al continuo desarrollo de las nuevas tecnologías".

Consecuentemente, al acceso a los superiores niveles de la Administración requiere determinados requisitos de formación académica, además de la adecuada adecuación técnica que solamente se obtiene tras la posesión de títulos universitarios que habilitan para el ejercicio de puestos específicos. Hay plazas que exigen haber cursado carreras técnicas de especial capacitación pericial y otras que admiten optar a las mismas desde diversas titulaciones o niveles académicos, según el carácter del cuerpo a cubrir y es normal que en el transcurso de su vida pública, un funcionario pase por diversas competencias y servicios, dentro de su mismo rango.

\section{La situación profesional del Protocolo como actividad específica}

La Escuela Internacional de Protocolo (EIP), a través de su Observatorio Profesional realizó (García: 2009) en su día el primer gran estudio sobre el estado de la profesión de los técnicos de protocolo, relaciones institucionales y organizadores de eventos que se realiza en el mundo. Publicado en la Revista Internacional de Protocolo sigue teniendo plena vigencia. Consistió en el análisis de 9.802 encuestas realizadas entre diferentes personas vinculadas de forma directa a este campo.

Resultado llamativo del estudio es que el sector privado representaría el 64,87 por ciento, frente al 35,13 por ciento del sector oficial. Analizando los datos relativos a la adscripción de profesionales al sector oficial, quedó de manifiesto que casi la mitad desarrollan su función en entidades locales, especialmente, en los ayuntamientos, que duplican a los servicios de protocolo vinculadas a las instituciones autonómicas.

Los estudios específicos de protocolo se imponen de forma arrolladora hasta los 30 años y que mantienen una posición de privilegio entre los 30 y los 50 años. Entre quienes estudiaron licenciaturas domina con claridad la rama de conocimiento de Ciencias Sociales y Jurídicas, y dentro de la misma, lo que más abundan son los estudios de Comunicación. 
Es evidente el poderoso dominio estadístico de la opción que combina el protocolo, la comunicación y las relaciones institucionales y, sin embargo, se considera sorprendente que los másteres específicos en protocolo apenas superen en un punto el 10 por ciento, lejos del 49 por ciento del generalista. Casi la mitad de los profesionales de protocolo comparten funciones con otros departamentos de forma continua o esporádica. El 73 por ciento de los encuestados se mostraron partidarios de la existencia de un carné profesional que acredite a quienes tengan estudios de grado o máster o experiencia laboral dilatada previa a la existencia de estudios universitarios.

\section{La controvertida coincidencia de salidas profesionales}

El simple cotejo de los vigentes planes de estudio y salidas profesionales de las dos Universidades (una privada y otra pública) que en estos momentos ofrecen el grado de Protocolo y Organización de Eventos nos depara la sorpresa de hallar, en ambos casos, repetidas coincidencias con salidas profesionales específicas que ya están cubiertas por otras carreras con mayor abolengo dentro de la familia de la Comunicación.

Cuando los egresados lleguen al mercado laboral van a tener que competir con los graduados en Periodismo y en Publicidad y Relaciones Públicas que han sido preparados específicamente para los mismos puestos que, con habilidad de mercaderes, se ha incluido en la misma bolsa de las salidas profesionales, no ya contra las directrices de la reforma universitaria, sino contra el más elemental sentido común.

Conviene contrastar los contenidos. Según la propia ANECA, la titulación de Publicidad y Relaciones Públicas está diseñada a partir de cuatro perfiles profesionales básicos y de cuatro bloques temáticos:

- Perfiles profesionales

A. Director/a de comunicación, investigador/a y consultor/a estratégico en publicidad y relaciones públicas.

- Profesionales que ejercen su actividad desde el ámbito de las organizaciones o bien desde la agencia de publicidad o de relaciones públicas y similares (cuentas y planificación estratégica, por ejemplo) o desde la empresa consultora.

- Responsables del área de comunicación, director de publicidad y de relaciones públicas, de un organismo o empresa, que establecen contacto con los diversos públicos potenciales, tanto internos como externos, así como de la planificación, gestión y control del plan de comunicación anual.

- Los investigadores y/o consultores estratégicos identifican el papel específico que ha de jugar la comunicación en cada organización y en las acciones de mercadotecnia. En ambos casos, definen las estrategias de comunicación de acuerdo con los objetivos de los emisores. Planifican tanto las investigaciones "ad hoc" necesarias para llegar a definir los ejes fundamentales de la campaña como el desarrollo e implantación de las propias campañas que supervisan, ejecutan y/o controlan.

Para ello cuentan con su conocimiento de los mercados y de las herramientas de comunicación, monitorización y control. Impartir formación en comunicación e investigar $\mathrm{y}$ formar docentes e investigadores para todos los niveles del sistema educativo. 
B. Investigadores/as, Planificadores/as y Compradores de medios

Estos profesionales planifican la idoneidad y características de los soportes para la difusión de las campañas de comunicación, así como el control y seguimiento de la audiencia de dichos medios y su eficacia, de acuerdo con los objetivos de comunicación definidos, con el fin de llegar a un público objetivo a través de los medios convencionales y no convencionales, mediante la adquisición y creación de espacios y soportes en las mejores condiciones posibles.

\section{Creativo/a y diseñador/a}

Profesional especializado en creatividad publicitaria en todo tipo de soportes, desde la conceptualización y visualización de la idea publicitaria hasta su adecuación e integración en los distintos medios. Se puede identificar las especialidades de: director creativo, director de arte, redactor y webmaster (creatividad de la estructura de la Web). La tarea de ejecutar hasta el arte final y controlar la producción física de los soportes corresponde a los expertos en producción y tráfico.

D. Gestor/a de comunicación corporativa

Profesional responsable de la gestión estratégica de la imagen y de la comunicación corporativa, tanto en su naturaleza intangible (identidad visual, comunicación y cultura corporativa) como en sus interrelaciones funcionales (financiera, comercial, de producción, etc.), y de establecer diálogos constructivos con los diferentes públicos relevantes de la empresa, internos y externos. También se ocupa de la reputación corporativa, en función del grado de cumplimiento de sus compromisos en relación con los públicos implicados.

Como señala Elena Real $(2005)^{3}$, puede afirmarse que los estudios de comunicación en España tienen una tradición, experiencia y solidez investigadora, académica y profesional que convierten el modelo universitario español en referencia para otros países europeos como Italia, Francia y Portugal. Y también advierte:

"Las nuevas titulaciones de grado deben diseñarse teniendo muy presente el perfil, las competencias y las destrezas profesionales que necesitan adquirir los futuros titulados. Además, no se podrán establecer títulos cuyos contenidos y efectos profesionales sean coincidentes, por lo que cada diploma deberá remitir en exclusiva a una determinada condición profesional. Será preciso, pues, conocer y concretar qué es un periodista, un publicitario, un profesional de las relaciones públicas, de la comunicación audiovisual, etc. Todos son comunicadores sociales, pero no todos hacen las mismas cosas ni comparten idénticas funciones comunicativas (que pueden ser parecidas pero no semejantes), y por ello requieren igualmente preparaciones académicas específicas, correctamente diferenciadas".

\section{Conclusiones}

Sin entrar a discutir el anhelo de los profesionales del Protocolo de disponer de una titulación específica, de rango universitario oficial; es evidente que el modo en que se

3 Para un análisis más pormenorizado de esta cuestión se puede consultar la tesis doctoral de la profesora Elena Real (2003), especialmente los capítulos II y III. 
ha articulado esta nueva carrera presenta numerosas coincidencias con los ya vigentes estudios de Relaciones Públicas e incluso de Periodismo. Pero donde las colisiones son más evidentes resulta ser en el espacio de las salidas profesionales.

Cierto que el mercado y la fuerza normativa de los hechos seleccionará en el futuro a los mejores o a los que tengan más suerte o fortuna; pero resulta llamativo que la ANECA haya autorizado la puesta en el mercado una nueva carrera con un diseño tan coincidente con otras ya establecidas y, sobre todo, conforme a los criterios del Plan Bolonia y de todos los documentos que lo desarrollan que ofrece las mismas salidas profesionales que ya atienden otros estudios específicos.

Se puede entender, desde una perspectiva comercial, que las dos universidades que ofrecen el grado de protocolo deseen hacerse con la mayor cuota de mercado ofreciendo tantas o más salidas para la carrera de Protocolo que cualquier otra, invadiendo sin recato otros espacios que se supone deben corresponder a otro tipo de estudios ya consolidados por una larga experiencia y contraste. Dicho de un modo popular: "Para ese viaje no se precisaban alforjas".

\section{Referencias bibliográficas}

ANECA (2005): Libro Blanco de los Títulos de Grado en Comunicación. Disponible en: http://www.aneca.es/media/150336/libroblanco_comunicacion_def.pdf

CANEL, María José; RODRÍGUEZ ANDRÉS, Roberto; y SÁNCHEZ ARANDA José Javier (2000): Periodistas al descubierto. Retrato de los profesionales de la información Madrid, CIS.

CONFERENCIA DE DECANOS DE INFORMACIÓN Y COMUNICACIÓN (2003): "Documentos europeos para la construcción del Espacio Europeo de Educación Superior". Sevilla, Universidad de Sevilla, noviembre.

ESCOBAR ROCA, Guillermo (2002): Estatuto de los periodistas. Régimen normativo de la profesión y organización de las empresas de comunicación. Madrid, Tecnos.

FERNÁNDEZ AREAL, Manuel (1977): Introducción al Derecho de la Información, Madrid, ATE.

GARCÍA, Ismael (2009): "Estado de la profesión de protocolo: primer macroestudio del Observatorio Profesional de la EIP". Madrid, Revista Internacional de Protocolo: "Ceremonial, Etiqueta, Heráldica, Nobiliaria y Vexilogía", ISSN 1135-9692, No. 50, pp. 42-51. Madrid.

MARTIN, A.: "Homologación europea", en Gaceta Complutense, 13 de noviembre de 2001, p. 7.

RAMOS FERNÁNDEZ, Fernando (1998): La profesión periodistica en España. Pontevedra, Servicio de Publicaciones de la Diputación Provincial de Pontevedra.

REAL RODRÍGUEZ, Elena (2003): Formación y ejercicio profesional del periodista en la España del siglo XXI dentro del marco de la Unión Europea. Tesis doctoral. Madrid, Facultad de Ciencias de la Información, Universidad Complutense de Madrid, noviembre. 
REAL RODRÍGUEZ, Elena (2009): "Interrogantes en torno a los estudios de Periodismo". CIC (Cuadernos de Información y Comunicación). 270 ISSN: 1135-7991.

REVISTA DE PROTOCOLO (2010): Monográfico, Número 56 (julio, agosto y septiembre): "Por fin título oficial". Madrid, Ediciones Protocolo.

VIVAR, Alberto y otros (2010): "Análisis de los estudios de comunicación en España frente al reto del Espacio Europeo de Educación Superior": http://www.aeic.org/malaga2010/upload/ok/307.pdf [Consultado: 5 de septiembre de 2012]

\section{Fernando RAMOS FERNÁNDEZ}

Universidad de Vigo

Facultad de Ciencias Sociales y de la Comunicación

Profesor titular de Periodismo

ferramos@telefonica.net 